\title{
BECAS Y PRÁCTICAS NO LABORALES COMO VÍA DE INSERCIÓN EN EL MERCADO LABORAL: ¿EXCLUSIÓN SOCIAL?
}

\author{
David Hernando Espada \\ Becario del Área de Estudios del Consejo Económico y Social de España
}

DOI: $10.1387 /$ lan-harremanak.15429

\section{ABSTRACT}

El recurso a las becas y prácticas no laborales parece que se ha convertido en el principal mecanismo de inserción en el mercado de trabajo que tienen los jóvenes. En este sentido, el Consejo de la Unión Europea en su Recomendación, de 10 de marzo de 2014, sobre un marco de calidad para los periodos de prácticas (traineeship) destaca la importancia de los mismos, ya que facilitan la transición del sistema educativo al mercado de trabajo. El presente trabajo tiene por objeto estudiar tales figuras, con la finalidad de determinar si este colectivo cuenta con la suficiente protección social o, si por el contrario, se está ante un colectivo caracterizado por la precariedad laboral. Se analizará, por un lado, las prácticas académicas y las prácticas no laborales, $y$, por otro lado, se estudia el sector de los becarios de investigación.

Palabras clave: Becas, prácticas no laborales, inserción, jóvenes, mercado de trabajo.

Turning to traineeships and internships seems to have become the main mechanism of inclusion into the job market for young people. According to this, the Council of the European Union, in its Council Recommendation of 10 March 2014 on a Quality Framework for Traineeships, underlines the importance of these periods, since they make easier the transition from the educational system to the job market. This paper aims to study such figures, in order to determine if this collective has 
enough protection or, on the contrary, if we are facing a group characterize by job insecurity. It will be analyzed, on the one hand, the traineeships and the internships, and on the other hand, it will be studied the collective of research scholars.

Key Words: Trineeship, internship, inclusion, young people, jos market. 


\section{Introducción}

El presente trabajo tiene por objeto analizar si se está ante el fenómeno de la precariedad laboral en dos sectores: por un lado, el constituido por los estudiantes universitarios y de formación profesional, que realizan prácticas en empresas y organismos públicos durante sus estudios, o una vez finalizados, y el colectivo de jóvenes de 18 a 25 ańos sin experiencia profesional, que pueden realizar las prácticas no laborales; y por otro, el sector de los becarios de investigación. En estas líneas no se aborda el estudio de otro colectivo que tradicionalmente se ha caracterizado por la precariedad de su relación, los médicos residentes, ya que tras la aprobación de la Ley 44/2003, de 21 de noviembre, de ordenación de las profesiones sanitarias, se estableció la laboralidad de la relación entre el médico residente y las entidades titulares docentes.

Los colectivos citados no se encuentran, en principio, dentro del ámbito de protección del Derecho del Trabajo y de la Seguridad Social, dado que no concurre en ellos las denominadas notas de laboralidad del art. 1.1 del Estatuto de los Trabajadores (en adelante ET): voluntariedad, retribución, dependencia y ajenidad, en concreto, la remuneración. El por qué de la exclusión se ha sustentado en que el elemento fundamental de las prácticas o becas es la formación, la remuneración que existe se entiende que es una asignación dineraria o en especie orientada a posibilitar el estudio y formación.

No obstante, en los últimos tiempos se le ha otorgado cierta protección social. Así, por un lado, los estudiantes universitarios y de formación profesional, y los jóvenes que realicen las prácticas no laborales, se asimilan a trabajadores por cuenta ajena a efectos de su inclusión en el Régimen General de la Seguridad Social (en adelante, RGSS), por lo que, acceden, en principio, a la acción protectora de la Seguridad Social establecida para este Régimen. Por otro lado, el sector de los becarios de investigación ha sufrido en los últimos años una consecutiva laboralización, por lo que, igualmente tienen, en principio, acceso a la protección social contemplada para el RGSS.

El Consejo de la Unión Europea, en su Recomendación de 10 de marzo de 2014, sobre un marco de calidad para los períodos de prácticas, ha señalado la importancia de los citados períodos, ya que se han convertido en un mecanismo 
que facilita la transición de los jóvenes del sistema educativo al mundo laboral. El desarrollo de las prácticas se ha de enmarcar, señala el Consejo, en un marco de calidad, ya que la capacidad de los períodos de prácticas para facilitar la entrada en el mercado laboral dependerá de la calidad del contenido didáctico y de las condiciones laborales que ofrezcan. En este sentido, recomienda a los Estados miembros, entre otros aspectos: que se garantice que se respetan los derechos y las condiciones laborales recogidos en la legislación nacional y europea aplicable; animar a las organizaciones que ofrecen prácticas especifiquen si ofrecen la cobertura del seguro de enfermedad y accidentes y de la baja por enfermedad, y garantizar una duración razonable de los períodos de prácticas que, en principio, no deben superar los seis meses, excepto en casos en que esté justificada una mayor duración.

La OCDE, en el estudio publicado en 2015 sobre habilidades y empleabilidad de los jóvenes, señala que las prácticas en empresas pueden facilitar la transición entre el ámbito académico y el mercado laboral. El estudio recoge los datos del eurobarómetro del año 2013, España es el segundo país europeo, por detrás de Eslovenia, donde más jóvenes trabajan en prácticas. De acuerdo con el citado indicador, en nuestro país el $67 \%$ de los jóvenes realiza un período de prácticas tras finalizar sus estudios, el 56\% desarrolla prácticas una sola vez, y un $11 \%$ vuelve a repetir la experiencia, por lo que parece que la citada situación no es irrelevante en nuestro país.

En este contexto, en las líneas que se siguen, se analizará el colectivo referido con la finalidad de determinar si cuenta con la suficiente protección social o, si por el contrario, se encuentra caracterizado por la precariedad laboral. Se estudia, por un lado, las prácticas académicas y las prácticas no laborales, y, por otro lado, se analiza el sector de los becarios de investigación.

\section{Prácticas académicas y prácticas no laborales}

Con el fin de concluir si existe precariedad laboral en relación a los colectivos mencionados en las líneas anteriores, se estudian a continuación: en primer lugar, las prácticas académicas, y en segundo lugar, las prácticas no laborales.

\section{Prácticas académicas}

La figura de las prácticas académicas se reguló por vez primera en nuestro ordenamiento a través del RD 1497/1981, de 19 de junio, sobre programas de cooperación educativa. La finalidad de la norma era reforzar la formación de los alumnos universitarios en empresas con el objetivo de preparar su incorporación al mercado de trabajo. En materia de protección social el texto legal se limitó a establecer que reglamentariamente se dictarían las normas oportunas para adap- 
tar el seguro escolar a un régimen especial para los alumnos que se encontraran realizando las prácticas externas. En este punto, Moreno Gené (Moreno, 2014) manifiesta que, pese a la interpretación que realizó la Tesorería General de la Seguridad Social, que permitía ampliar la cobertura del seguro escolar a estos supuestos de prácticas externas, esta solución resultaba muy limitada a la hora de dotar de una adecuada protección social, ya que las prestaciones derivadas del seguro escolar se circunscribían a accidente escolar, enfermedad e infortunio familiar.

La cuestión sobre la adecuada protección social de los estudiantes que desarrollan prácticas académicas no se aborda hasta fechas recientes. La Ley 27/2011, de 1 de agosto, sobre actualización, adecuación y modernización del sistema de Seguridad Social, en su disposición adicional tercera, ordenó al Gobierno que en el plazo de tres meses desde la publicación de la Ley, en base a las previsiones contenidas en el art. 97.2.m) LGSS y en los términos y condiciones determinados reglamentariamente, estableciera los mecanismos de inclusión de los participantes en programas de formación financiados por organismos o entidades públicos o privados, los cuales, vinculados a estudios universitarios o de formación profesional, conllevaran contraprestación económica para los afectados, siempre que en razón de la realización de dichos programas, y conforme a las disposiciones en vigor, no viniesen obligados a estar de alta en el correspondiente Régimen de la Seguridad Social.

El desarrollo de la referida disposición adicional tercera, viene dado por el RD 1493/2011, de 24 de octubre, disponiendo, en su art. 1, la asimilación a trabajadores por cuenta ajena, siempre y cuando se den los requisitos anteriormente señalados. En relación a la acción protectora, se establece que es la correspondiente al RGSS, pero excluyendo la protección por desempleo. No obstante, poco después el Gobierno aprueba el RD 1707/2011, de 18 de noviembre, por el que se regulan las prácticas académicas externas de los estudiantes universitarios, donde a través de su disposición adicional primera se excluía a dichos estudiantes del ámbito de aplicación de la Seguridad Social.

Ante dicha exclusión Comisiones Obreras interpuso recurso contenciosoadministrativo con el Real Decreto. El Tribunal Supremo, Sala de lo Contencioso-Administrativo, resolvió la controversia planteada a través de su sentencia de 21 de mayo de 2013 (rec. 171/2012). La Sala declara la nulidad de la norma reglamentaria sin entrar en el fondo del asunto, ya que la incorporación de la disposición adicional primera al texto legal no se remitió al Consejo de Estado para que se pronunciase sobre la misma, al tratarse de una disposición sustancial y relevante, el Gobierno debía conocer la opinión del referido órgano consultivo, de conformidad a lo dispuesto en la Ley Orgánica 3/1980, de 22 de abril, reguladora del Consejo de Estado. En este contexto, se volvió a la aplicación del RD 1497/1981 mientras que el Gobierno elaboraba una nueva disposición. El 
nuevo reglamento se aprobó el pasado año, el RD 592/2014, de 11 de julio, que no prevé disposición alguna contraria a la asimilación a trabajador por cuenta ajena, a efectos de inclusión en el RGSS, de los estudiantes universitarios que realizan prácticas externas.

\section{Prácticas no laborales}

El RD 1543/2011, de 31 de octubre, regula las prácticas no laborales en empresas. La norma se dicta a consecuencia del alto nivel de desempleo juvenil provocado por la crisis económica; según el RD, los jóvenes encuentran una especial dificultad para insertarse en el mercado laboral. Estos jóvenes, tienen formación académica acreditada, sin embargo, carecen de la experiencia profesional necesaria. Dichas prácticas las pueden desarrollar jóvenes de entre 18 y 25 años, desempleados, que tengan cualificación pero nula o escasa experiencia profesional, en empresas durante un período de entre tres y nueve meses. La inclusión de estos jóvenes en la Seguridad Social se realiza de conformidad al RD 1493/2011, esto es, en los mismos términos que para los estudiantes que realizan prácticas académicas.

\section{3. ¿Acción protectora? Modulación respecto al trabajador por cuenta ajena}

La acción protectora, tal y como se ha expuesto ut supra, es la correspondiente al RGSS. No obstante, esta es objeto de modulaciones respecto del trabajador por cuenta ajena, siendo la más destacada la exclusión de la protección por desempleo. La justificación de tal omisión se halla en que la prestación por desempleo es sustitutiva de rentas salariales. En consecuencia, como señala la jurisprudencia (véase STS, Sala de lo Contencioso-Administrativo, 28 junio 2005, rec. 150/2003), en los supuestos aquí estudiados no existe derecho a tal protección, ya que la cuantía percibida en el desarrollo de las prácticas no posee naturaleza salarial.

La cotización de este colectivo también encuentra matices respecto de las reglas aplicables a los trabajadores por cuenta ajena. Las reglas de cotización son las previstas para el contrato de formación y aprendizaje. No obstante, los estudiantes y jóvenes aquí estudiados no cotizan por la contingencia de desempleo, ni por Fondo de Garantía Salarial, ni por formación profesional. La Orden ESS/86/2015, de 30 de enero, por la que se desarrollan las normas legales de cotización a la Seguridad Social, desempleo, protección por cese de actividad, Fondo de Garantía Salarial y formación profesional, contenidas en la Ley 36/2014, de 26 de diciembre, de Presupuestos Generales para el Estado para el ańo 2015, establece en su art. 44 la cotización en los contratos para la formación y el aprendizaje. La cotización a la Seguridad Social consistirá en una cuota mensual de 36,79 euros por contingencias comunes, de los que 30,67 euros serán a cargo del empresario y 6,12 euros a cargo del trabajador, y de 4,22 euros 
por contingencias profesionales, a cargo del empresario. La cuantía total a ingresar sería de 41,01 euros, de los que 34,89 euros corresponden al empresario y 6,12 euros al trabajador. En definitiva, esta será la cotización a la Seguridad Social correspondiente a los estudiantes universitarios y de formación profesional que desarrollen prácticas externas, así como del colectivo de jóvenes que realicen las llamadas prácticas no laborales.

En este punto, se ha de tener presente lo dispuesto en la Ley 18/2014, de 15 de octubre, de aprobación de medidas urgentes para el crecimiento, la competitividad y la eficiencia, en su disposición adicional vigesimosexta se ha previsto que las prácticas curriculares externas realizadas por los estudiantes universitarios y de formación profesional tienen una bonificación del $100 \%$ en la cotización a la Seguridad Social desde el día 1 de agosto de 2014, por lo que con esta medida se eliminan los costes que venían sufriendo empresas y organismos públicos desde la inclusión de los estudiantes en el RGSS, aunque tal bonificación no comprende las prácticas extracurriculares ni las denominadas prácticas no laborales.

En definitiva, conforme a lo expuesto, parece que se puede afirmar que en los últimos años se ha producido una normalización en la realización de las prácticas curriculares o extracurriculares de los estudiantes universitarios y de formación profesional. Sin embargo, la modulación de la acción protectora y de las reglas de cotización parece que permite sostener que se trata de un colectivo sometido a la precariedad laboral y, en consecuencia, traer consigo un mayor riesgo de exclusión social. Este riesgo vendría dado, principalmente, en la exclusión de la protección por desempleo, cuya justificación parece que se encuentra en la interpretación restrictiva que se ha producido por parte de la jurisprudencia. Esta postura se ha de mantener respecto de las prácticas no laborales establecidas en el año 2011 como instrumento para frenar el desempleo juvenil. En este caso, cabe plantearse el por qué no se adoptaron otro tipo de medidas que no crearan otra vía de precariedad. Finalmente, en relación a las reglas de cotización puede resultar cuestionable, por un lado, el que no se cotice por formación profesional, ya que la formación es la razón de ser de las prácticas $\mathrm{y}$, por otro lado, la cotización por contingencias comunes y profesionales es fija, con independencia del importe real de la beca o ayuda que se perciba.

\section{El sector de la investigación}

El sector de la investigación ha constituido en nuestro país uno de los ámbitos que tradicionalmente se ha caracterizado por la precariedad laboral del personal investigador, principalmente, en las primeras etapas de la carrera investigadora. Sin embargo, parece que en los últimos tiempos tal situación desaparece 
con la aprobación de la Ley 14/2011, de 1 de junio, de la Ciencia, la Tecnología y la Innovación (en adelante LCTI).

\section{La Ley $14 / 2011$, de 1 de junio, de la Ciencia, la Tecnología y la Innovación: ¿el fin de la precariedad en el ámbito de la investigación?}

La LCTI comporta, según Moreno Gené (Moreno, 2011), el final del estado de excepción laboral en la ciencia, ya que supone la eliminación de las becas de investigación en el ámbito de la ciencia, la tecnología y la innovación, en beneficio de la contratación laboral y, por tanto, la extensión de la protección laboral y social dispensada por el ordenamiento jurídico. En este mismo sentido, Serrano Argüello (Serrano, 2014), ha señalado que la renovada Ley de la Ciencia quiere postergar el anterior sistema de reclutamiento de los investigadores mediante becas (pre y postdoctorales), dado que éstas han supuesto un apartamiento del reconocimiento de la laboralidad y de una ausencia de protección de los derechos de Seguridad Social.

En efecto, la LCTI establece a que modalidades de contrato de trabajo puede acceder el personal investigador: contrato predoctoral, contrato de acceso al Sistema Español de Ciencia, Tecnología e Innovación, y contrato de investigador distinguido (art. 20). El régimen jurídico aplicable a estas modalidades contractuales es el previsto en la propia LCTI y en sus normas de desarrollo, y con carácter supletorio se estará a lo dispuesto en el Estatuto de los Trabajadores y en su respectiva normativa de desarrollo. En las líneas que se suceden se realiza una breve descripción sobre cada uno de los contratos anteriormente señalados.

a) El contrato predoctoral (art. 21), tiene como objeto la realización de tareas de investigación, en el ámbito de un proyecto específico y novedoso, por quienes estén en posesión del título de licenciado, ingeniero, arquitecto, graduado universitario con grado de al menos 300 créditos ECTS o máster universitario, o equivalente, y hayan sido admitidos a un programa de doctorado. Este personal tendrá la consideración de personal investigador predoctoral en formación.

El contrato se celebrará por escrito entre el investigador, en su condición de trabajador, y la universidad pública u organismo de investigación titular de la unidad investigadora, en su condición de empleador, con dedicación a tiempo completo. El contrato es de duración determinada, será de un año, prorrogable por períodos anuales previo informe favorable de la comisión académica del programa de doctorado, o en su caso de la escuela de doctorado, durante el tiempo que dure su permanencia en el programa. En ningún caso la duración acumulada del contrato inicial más las prórrogas podrán exceder de cuatro años. En este punto, se ha de matizar, por un lado, que cuando el contrato se concierte con una persona con discapacidad la duración máxima podrá alcanzar los seis ańos, por otro lado, las situaciones de incapacidad temporal, riesgo durante 
el embarazo, maternidad, adopción o acogimiento, riesgo durante la lactancia y paternidad, suspenderán el cómputo de la duración del contrato.

Finalmente, destacar que la retribución de este contrato no podrá ser inferior al $56 \%$ del salario fijado para las categorías equivalentes en los convenios colectivos de su ámbito de aplicación durante los dos primeros ańos, al $60 \%$ durante el tercer año, y al $75 \%$ durante el cuarto año. Tampoco podrá ser inferior al salario mínimo interprofesional que se establezca cada año.

b) El contrato de acceso al Sistema Español de Ciencia, Tecnología e Innovación (art. 22) podrá concertarse con quienes estén en posesión del título de doctor o equivalente, sin que sean de aplicación los límites de cinco ańos, o de siete años cuando el contrato se concierte con un trabajador con discapacidad, a que se refiere el art. 11.1 del Estatuto de los Trabajadores (contrato de trabajo en prácticas). El trabajo a desarrollar consistirá, principalmente, en la realización de tareas de investigación, orientadas a la obtención por el personal investigador de un elevado nivel de perfeccionamiento y especialización profesional, que conduzcan a la consolidación de su experiencia profesional.

Respecto a su duración, no podrá ser inferior a un año, ni exceder de cinco años. Cuando el contrato se hubiese concertado por una duración inferior a cinco ańos podrá prorrogarse sucesivamente sin que, en ningún caso, las prórrogas puedan tener una duración inferior al año. Asimismo, tal y como sucede en el contrato predoctoral, las situaciones de incapacidad temporal, riesgo durante el embarazo, maternidad, adopción o acogimiento, riesgo durante la lactancia y paternidad, suspenderán el cómputo de la duración del contrato. Por último, señalar que la retribución de este contrato no podrá ser inferior a la que corresponda al personal investigador que realice actividades análogas.

c) El contrato de investigador distinguido (art. 23) se podrá celebrar con investigadores españoles o extranjeros de reconocido prestigio en el ámbito científico y técnico, que se encuentren en posesión del título de doctor o equivalente. De esta modalidad se puede destacar, que su objeto será la realización de actividades de investigación, instalaciones y programas científicos y tecnológicos singulares de gran relevancia en el ámbito de conocimiento de que se trate, en el marco de las funciones y objetivos del empleador, y el contrato tendrá la duración que las partes acuerden.

A la luz de lo expuesto, parece que puede afirmarse, en la línea de lo sostenido por la doctrina citada "ut supra», que se supera la precariedad laboral a la que sometía la legislación, en términos generales, al personal investigador. Si se realiza un análisis a la normativa anterior a la LCTI se encuentra la justificación a la afirmación sostenida. La primera intervención legislativa se produce con el RD 1326/2003, de 24 de octubre, por el que se aprueba el Estatuto del Becario de Investigación (en adelante EB), su objetivo fue el de dotar al colectivo de 
becarios de investigación de una protección social adecuada, dado que con anterioridad a la aprobación del EB el personal investigador carecía de una regulación legal o reglamentaria más allá de las normas específicas de cada convocatoria. Así, en orden a cumplir tal fin el EB incluyó al becario de investigación en el EB, inclusión realizada vía art. 97.2.l) LGSS.

Sin embargo, el fin del EB terminó por resultar ineficaz ante los requisitos que se debían cumplir a efectos de acceder al RGSS. La condición de becario de investigación concurría en aquellos titulados universitarios que fuesen beneficiarios de una beca concedida en virtud de programas inscritos en un Registro de becas de investigación (creado a estos efectos) y, además, estos titulados debían estar en posesión del título de doctor o haber obtenido el reconocimiento de su suficiencia investigadora (Diploma de Estudios Avanzados, DEA). La doctrina fue crítica en torno a la exigencia de tales requisitos (Morón, 2006, y Cristóbal, 2008). En este punto, Moreno Gené (Moreno, 2006) señaló: por un lado, que la limitación de la protección social a los investigadores predoctorales con el DEA y a los postdoctorales no justificaba el tratamiento diferenciado respecto de los becarios que no habían obtenido reconocimiento de su suficiencia investigadora y, por otro lado, la inscripción en el citado Registro se dejaba a la voluntad de las entidades que convocaban las becas de investigación no estableciendo ninguna obligación expresa al respecto. No obstante, pese a las críticas de la doctrina, la Sala de lo Contencioso-Administrativo del Tribunal Supremo consideró razonables los requisitos establecidos por el EB (véase SSTS 28 junio 2005, rec. 150/2003, y 18 noviembre 2005, rec. 1/2004).

Respecto a la acción protectora y la cotización, el EB estableció que la acción protectora sería la correspondiente al RGSS, con la única excepción de la protección por desempleo. En relación a la cotización se aplicarían las normas comunes del Régimen General, pero se debía atender a una serie de reglas específicas, entre ellas, la base de cotización, tanto por contingencias comunes como profesionales, estaría constituida por la cuantía del tope mínimo absoluto vigente en cada momento en el RGSS, y no existía la obligación de cotizar respecto a la contingencia de desempleo, Fondo de Garantía Salarial, ni por formación profesional. La introducción de dichas reglas no carecía de importancia, ya que, de un lado, la mínima base de cotización incidía en el quantum de las prestaciones futuras y, por otro lado, la exclusión de la protección por desempleo resultaba controvertida.

Las críticas vertidas al EB y, pese a su convalidación por la jurisprudencia, provocaron que el Gobierno dictara el RD 63/2006, de 27 de enero, por el que se aprueba el Estatuto del Personal Investigador en Formación (en adelante EPIF). El EPIF introduce un nuevo concepto de personal investigador en formación, tendrán esta condición aquellos graduados universitarios que sean beneficiarios de programas de ayuda dirigidos al desarrollo de actividades de 
formación y especialización científica y técnica a través, como mínimo, de los correspondientes estudios oficiales de doctorado, siendo de aplicación a cualquier programa, con independencia de la naturaleza pública o privada de la entidad convocante. En definitiva, se produce una ampliación del ámbito subjetivo. No obstante, puede señalarse que la principal novedad del EPIF es el denominado "modelo $2+2$ ", es decir, un período inicial de dos años de beca de investigación finalizando con dos años mediante un contrato de trabajo. La doctrina, en términos generales, alabó la aprobación del EPIF, aunque matizó que se trataba de un "tímido paso en la reordenación de la actividad formativa e investigadora» (Morón, 2006, y Cristóbal, 2008).

En relación a la acción protectora y la cotización, respecto del personal de beca, se establece que la acción protectora, al igual que en el EB, es la correspondiente al RGSS con la excepción de la protección por desempleo. Respecto a la cotización es la base mínima de cotización vigente en cada momento para el grupo de cotización 1 (ingenieros y licenciados), siendo la misma tanto para las contingencias comunes como para las profesionales ${ }^{1}$. Asimismo, tal y como recogía el EB, no existe obligación de cotizar por la contingencia de desempleo, al Fondo de Garantía Salarial, ni por formación profesional. Esta situación en los dos primeros años en el investigador en formación, pese a que la aprobación del EPIF fue alabada, se criticó por parte de la doctrina, destacando Moreno Gené (Moreno, 2006) que la inclusión del personal investigador en formación de beca en el RGSS se realizaba de forma "debilitada»: por un lado, la exclusión de protección por desempleo y, por otro, en relación a la base de cotización se señalaba la preferencia de tener en cuenta el importe de las cuantías efectivamente percibidas por el investigador, dado que con carácter general eran superiores a la base de cotización única fijada por el EPIF.

En virtud de lo señalado parece obvio que la LCTI no ha supuesto un tímido paso en su apuesta por la laboralización del vínculo que une al personal investigador con los distintos organismos, principalmente en los primeros años de formación del personal investigador. No obstante, en el momento en que se redactan las presentes líneas no puede decirse que la referida laboralización se haya materializado de una manera efectiva. La LCTI, en su disposición adicional segunda, establece que en el plazo de dos años desde la entrada en vigor de la Ley, el Gobierno elaborará un estatuto del personal investigador en formación. Dicho estatuto sustituirá al EPIF, e incluirá las prescripciones recogidas en la LCTI para el contrato predoctoral. Esta disposición ha de leerse junto con su disposición final undécima, la LCTI entrará en vigor a los seis meses

${ }^{1}$ El EPIF estableció para el año 2006 que la base de cotización, tanto por contingencias comunes como profesionales, estaría constituida por la cuantía del tope mínimo absoluto de cotización vigente en cada momento en el RGSS. La norma acudió a esta fórmula, que ya recogía el Estatuto del Becario de Investigación, de manera provisional por razones presupuestarias. 
de su publicación en el BOE, a excepción, entre otras disposiciones, del contrato predoctoral, entrando éste en vigor al año de la publicación de la Ley en el BOE.

El Gobierno ha incumplido el mandato del legislador, por lo que aún se encuentra en vigor el EPIF. En este punto, se ha de hacer referencia que la LCTI, en su disposición transitoria cuarta, establece que los programas de ayuda al personal investigador en formación financiados con fondos públicos, incluidos en el ámbito de aplicación del EPIF, existentes a la entrada en vigor del contrato predoctoral que regula la LCTI, deberán adaptarse a lo dispuesto para dicho contrato únicamente por lo que respecta a las convocatorias que se publiquen a partir de ese momento. No obstante, para las convocatorias que se encuentren en ejecución a la entrada en vigor, continuará vigente el «modelo $2+2$ ». Ante el citado incumplimiento cabe plantearse qué sucede, en concreto, con el contrato predoctoral. En este sentido, se ha manifestado que la LCTI se aplique, ya que es «evidente que no era intención del legislador mantener esta situación sine die» (Duque, 2014).

El presente trabajo ha de finalizar señalando que la LCTI supone un cambio significativo en el sector de la investigación, sin duda, positivo. No obstante, la regulación del contrato predoctoral aún parece algo precaria, pronunciándose en este mismo sentido Serrano Argüello (Serrano, 2014). La nota de precariedad debe destacarse respecto del régimen retributivo que se establece para los investigadores que accedan al referido contrato: una retribución mínima del $56 \%$ del salario fijado para las categorías equivalentes en el convenio colectivo durante los dos primeros ańos y del 60 y $75 \%$ durante el tercer y cuarto año. En este punto, Moreno Gené (Moreno, 2011) señala, que podría darse la situación de que con contrato predoctoral los investigadores perciban retribuciones inferiores a las percibidas como becarios. Por tanto, en este contexto, parece que una solución sería la de adoptar el régimen retributivo que el ET establece para el contrato en prácticas, la retribución no podrá ser inferior al 60 o al $75 \%$ durante el primero o el segundo año de vigencia del contrato.

\section{Consideraciones finales. Posibles medidas contra la precariedad}

En el presente trabajo se ha planteado si la precariedad laboral caracteriza, por un lado, las prácticas que desarrollan los estudiantes universitarios y de formación profesional durante sus estudios, o tras finalizar estos, así como en las prácticas no laborales que pueden realizar los jóvenes entre 18 y 25 ańos; y, por otro lado, al sector de la investigación, en concreto en el contrato predoctoral. En las sucesivas líneas, de conformidad al análisis realizado, se efectúan una serie de valoraciones finales en relación a los dos colectivos señalados. 
Los períodos de prácticas desarrollados por los estudiantes universitarios o de formación profesional durante su formación, o tras graduarse, así como en el caso de los jóvenes que realizan las prácticas no laborales, parecen caracterizarse, en términos generales por su precariedad. La inclusión en el RGSS, mediante su asimilación a trabajadores por cuenta ajena ha de valorarse positivamente. Sin embargo, dicha valoración, puede cuestionarse por dos factores: por un lado, la protección por desempleo se excluye de la acción protectora y, por otro lado, respecto de las reglas de cotización adoptadas para este colectivo. En definitiva, parece que no se está ante la verdadera protección del Derecho del Trabajo y de la Seguridad Social.

El Consejo de la Unión Europea, en su Recomendación de 10 de marzo de 2014, considera que en las últimas dos décadas los períodos de prácticas se han convertido es una importante puerta de entrada al mercado laboral, ya que facilita la transición del sistema educativo al mercado de trabajo, por lo tanto, parece que el Derecho del Trabajo debería incluir a este colectivo en su ámbito de aplicación, garantizando así, unas prácticas de calidad. La OCDE en su estudio sobre jóvenes y empleabilidad de 2015, igualmente, refleja la importancia de las prácticas como mecanismo de inserción en el mercado de trabajo. No obstante, dicho estudio deja de manifiesto que en nuestro país el uso de las prácticas o becas puede llegar a considerarse abusivo. Dicha circunstancia puede manifestar, por un lado, una generalizada situación de precariedad laboral, y por otro lado, cabe plantearse si existe una efectiva inserción en el mercado de trabajo tras un período de prácticas, ya que existe un porcentaje de jóvenes en nuestro país que repiten la experiencia.

En este contexto, sería adecuado establecer una duración a los períodos de prácticas. El Consejo ha señalado, en la Recomendación referida, que dichos períodos deberían de tener una duración razonable, no superar los seis meses, excepto en casos en que esté justificada una duración mayor. En este sentido, la regulación del contrato de trabajo en prácticas previsto en el art. 11.1 ET, establece que la duración del contrato no podrá ser inferior a seis meses ni exceder de dos años. Ningún trabajador podrá ser contratado en prácticas en la misma o distinta empresa por tiempo superior a dos ańos en virtud de la misma titulación o certificado de profesionalidad. El citado contrato, se puede concertar con quienes estén en posesión de un título universitario o de formación profesional, no obstante, los límites que establece podrían ser orientativos de cara a establecerlos en los períodos de prácticas que se desarrollan durante los estudios por parte de alumnos universitarios y de formación profesional.

En virtud de lo expuesto en líneas anteriores, parece que los periodos de prácticas o becas deberían considerarse como parte de la formación del alumno y por lo tanto, ceńirse a un estado previo a la consecución del título. Este tipo de becas y prácticas deben considerarse como un mecanismo para facilitar el ac- 
ceso al mercado de trabajo una vez finalizado el periodo de formación. Los jóvenes posgraduados, deberían acceder directamente a un contrato de trabajo de prácticas. Así, la "Estrategia de Emprendimiento y Empleo Joven 2013-2016», podría tener un mayor alcance, es decir, las medidas destinadas a incentivar la inserción de los jóvenes en el mercado laboral serían más eficaces, entre ellas, los incentivos para los contratos de trabajo de prácticas.

Finalmente, respecto al sector de la investigación señalar que desde el año 2011 se puede decir que existe, en principio, una laboralización del personal investigador. Sin embargo, no puede afirmarse que dicha laboralización se halla aún materializado. El Gobierno ha incumplido el mandato de la LCTI sobre la elaboración de un nuevo estatuto del personal investigador que recogiera el nuevo contrato predoctoral. Además, pese a que hay que valorar de manera positiva esta normalización del personal investigador, parece que el contrato predoctoral establece un régimen jurídico con tintes de precariedad en la relación laboral, tal y como se apuntó, con el régimen retributivo: una retribución mínima del 56\% del salario fijado para las categorías equivalentes en el convenio colectivo durante los dos primeros ańos y del 60 y $75 \%$ durante el tercer y cuarto año.

En este punto, cabe plantearse la posibilidad de establecer un régimen retributivo distinto. Por un lado, a efectos orientativos se podría considerar el previsto para el contrato de trabajo de prácticas, se contempla que la retribución no puede ser inferior al 60 o $75 \%$ durante el primer o segundo ańo de vigencia del contrato, respectivamente, del salario fijado en convenio para un trabajador que desempeñe el mismo o equivalente puesto. Por tanto, podría ser adecuado utilizar este régimen retributivo para el investigador predoctoral, teniendo presente que la duración del contrato predoctoral es de cuatro años.

\section{Bibliografía}

Aragón Gómez, Cristina (2013): «La prestación contributiva de Seguridad Social», Pamplona, Lex Nova.

Barba Ramos, Francisco (2014): «La delgada línea entre la inserción y la explotación laboral. La esquizofrénica regulación de las prácticas universitarias», Monereo Pérez, José Luis (director), Retos del Derecho del Trabajo frente al desempleo juvenil. XXXII Jornadas Universitarias Andaluzas de Derecho del Trabajo y Relaciones Laborales, Consejo Andaluz de Relaciones Laborales, 2014, pp. 307-317.

Cristóbal Roncero, María del Rosario (2008): «El Estatuto Jurídico del Personal Investigador en Formación", Cizur Menor, Thomson Civitas.

Duque González, Mario (2014): «Análisis actual de la normativa de becarios, formación práctica y contratación de jóvenes», Revista de Información Laboral, n.o 2, pp. 81-108.

González Díaz, Francisco A., Cristóbal Roncero, María del Rosario (2011): «El personal investigador en formación», Sempere Navarro, Antonio V. (director), Relacio- 
nes laborales especiales y contratos con particularidades, Pamplona, Aranzadi, pp. 10131081.

GonzÁlez Ortega, Santiago (1999): «Las becas: ¿formación, inserción, prácticas profesionales, trabajo asalariado?», Cruz Villalón, Jesús (director), Trabajo subordinado y trabajo autónomo en la delimitación de fronteras del Derecho del Trabajo. Estudios en homenaje al profesor José Cabrera Bazán, Madrid, Tecnos, pp. 123-135.

Moreno Gené, Josep (2014): «La inclusión «definitiva» en la Seguridad Social de los estudiantes universitarios en prácticas», Estudios financieros. Revista de trabajo y seguridad social: Comentarios, casos prácticos: recursos humanos, n. ${ }^{\circ} 379$, pp. 157-202.

Moreno Gené, Josep (2012): «La regulación de las prácticas no laborales en empresas en el Real Decreto 1543/2011: ¿un sucedáneo de contrato en prácticas?», Estudios financieros. Revista de trabajo y seguridad social: Comentarios, casos prácticos: recursos humanos, n. 347 , pp. 77-118.

Moreno Gené, Josep (2011): «La contratación laboral de los investigadores en la Ley de la Ciencia, la Tecnología y la Innovación: ¡el final del estado de excepción laboral en la ciencia?», Estudios financieros. Revista de trabajo y seguridad social: Comentarios, casos prácticos: recursos humanos, n. ${ }^{\circ} 340$, pp. 79-124.

Moreno Gené, Josep (2006): «La Seguridad Social de los investigadores en formación. A propósito del nuevo estatuto del personal investigador en formación», Tribuna Social: Revista de seguridad social y laboral, n. ${ }^{\circ} 188-189$, pp. 22-48.

Morón Prieto, Ricardo (2006): «El Estatuto del Personal Investigador en Formación: otro tímido paso en la asimilación de las becas formativas al régimen laboral», Relaciones laborales: Revista crítica de teoría y práctica, n. ${ }^{\circ}$ 2, pp. 447-481.

Quesada Segura, Rosa (2004): «Los principios constitucionales y el modelo legal de protección por desempleo», Sevilla, Temas Laborales.

SÁnchez-Rodas Navarro, Cristina (2014): «Formas no laborales de inserción de los jóvenes en el mercado de trabajo», Monereo Pérez, José Luis (director), Retos del Derecho del Trabajo frente al desempleo juvenil. XXXII Jornadas Universitarias Andaluzas de Derecho del Trabajo y Relaciones Laborales, Consejo Andaluz de Relaciones Laborales, 2014, pp. 173-217.

Serrano ArgüEllo, Noemí (2014): «Modalidades específicas de contratación laboral del personal investigador. De la precarización al desiderátum de su estabilidad en el empleo y consolidación profesional», Revista General de Derecho del Trabajo y de la Seguridad Social, n. ${ }^{\circ}$ 36, pp. 131-174.

Serrano Argüello, Noemí (2013): «Los contratos laborales del personal investigador», Calonge Velázquez, Antonio (director), Ciencia, tecnología e innovación: nuevo régimen jurídico, Granada, Comares, pp. 85-116.

Todolí Signes, Adrían (2015): «La compleja e insatisfactoria regulación de las prácticas y las becas de trabajo, Trabajo y Derecho, n. ${ }^{\circ}$ 6, 2015, pp. 61-80. 